\title{
Der Weltklimarat IPCC/IAC
}

Der 1988 gegründete Weltklimarat Intergovernmental Panel on Climate Change (IPCC) und der Inter Academy Council (IAC) sind zwei zwischenstaatliche Institutionen für Klimawandel. Sie wurden vom Umweltprogramm der Vereinten Nationen (UNEP) und der Organisation für Meteorologie (WMO) ins Leben gerufen. Die IPCC überprüft und bewertet laufend die neuesten wissenschaftlichen, technischen und sozioökonomischen Informationen, die weltweit für das Verständnis des Klimawandels relevant sind. Sie führt keine Forschung durch und überwacht auch keine klimarelevanten Daten oder Parameter. Sie verschafft der Welt einen wissenschaftlichen Überblick über den aktuellen Wissensstand und die möglichen ökologischen und sozio-ökonomischen Auswirkungen des Klimawandels, ohne jedoch konkrete Lösungswege oder politische Handlungsweisung vorzuschlagen. Deshalb verliert ihre wertvolle Arbeit jegliche Kompetenz und verkommt zum einsamen Rufer in der Wüste.

Eine mögliche Lösung des Dilemmas bestünde darin, wenn die IPCC von den Vereinten Nationen (UNCFFF) dazu ermächtigt würde, die geopolitische Steuerung von Umwelt- und Klimafragen in allen Ländern der Erde zu übernehmen und eine neue Verfassung schreibt. Doch noch ist die IPCC von keiner Nation dazu legitimiert.

Selbst die UNO kann auf Basis ihrer aktuellen völkerrechtlichen Verfassung im Hinblick auf die drängenden Klima- und Umweltfragen nicht handeln, sondern nur beraten. Die Resolution der UN-Generalversammlung vom 24. September 2020 listet erneut bekannte Massnahmen und Absichtserklärungen zur Korrektur auf unserem Planeten auf. Doch es fehlen die rechtlichen Mittel, damit umwelt- und klimaorientierte Schwerpunkte verfasst werden und weltweit umgesetzt werden. 